\title{
Evidence of a liver-alpha cell axis in humans: hepatic insulin resistance attenuates relationship between fasting plasma glucagon and glucagonotropic amino acids
}

\author{
Nicolai J. Wewer Albrechtsen ${ }^{1,2} \cdot$ Kristine Færch $^{3} \cdot$ Troels M. Jensen $^{3} \cdot$ Daniel R. Witte $^{4,5} \cdot$ Jens Pedersen $^{1,2}$. \\ Yuvaraj Mahendran $^{2}$ - Anna E. Jonsson ${ }^{2}$. Katrine D. Galsgaard ${ }^{1,2}$. Marie Winther-Sørensen ${ }^{1,2}$. Signe S. Torekov ${ }^{1,2}$. \\ Torsten Lauritzen $^{5}$. Oluf Pedersen ${ }^{2}$. Filip K. Knop ${ }^{2,6,7}$ - Torben Hansen ${ }^{2}$ - Marit E. Jørgensen ${ }^{3,8}$ - Dorte Vistisen ${ }^{3}$. \\ Jens J. Holst ${ }^{1,2}$
}

Received: 4 July 2017 / Accepted: 23 November 2017 / Published online: 5 January 2018

(C) Springer-Verlag GmbH Germany, part of Springer Nature 2018

\begin{abstract}
Aims/hypothesis The secretion of glucagon is controlled by blood glucose and inappropriate secretion of glucagon contributes to hyperglycaemia in diabetes. Besides its role in glucose regulation, glucagon regulates amino acid metabolism in hepatocytes by increasing ureagenesis. Disruption of this mechanism causes hyperaminoacidaemia, which in turn increases glucagon secretion. We hypothesised that hepatic insulin resistance (secondary to hepatic steatosis) via defective glucagon signalling/glucagon resistance would lead to impaired ureagenesis and, hence, increased plasma concentrations of glucagonotropic amino acids and, subsequently, glucagon. Methods To examine the association between glucagon and amino acids, and to explore whether this relationship was modified by hepatic insulin resistance, we studied a well-characterised cohort of 1408 individuals with normal and impaired glucose regulation. In this cohort, we have previously reported insulin resistance to be accompanied by increased plasma concentrations of glucagon. We now measure plasma levels of amino acids in the same cohort. HOMA-IR was calculated as a marker of hepatic insulin resistance. Results Fasting levels of glucagonotropic amino acids and glucagon were significantly and inversely associated in linear regression models (persisting after adjustment for age, sex and BMI). Increasing levels of hepatic, but not peripheral insulin resistance $(p>0.166)$ attenuated the association between glucagon and circulating levels of alanine, glutamine and tyrosine, and was significantly associated with hyperaminoacidaemia and hyperglucagonaemia. A doubling of the calculated glucagon-alanine index was significantly associated with a 30\% increase in hepatic insulin resistance, a $7 \%$ increase in plasma alanine aminotransferase levels, and a $14 \%$ increase in plasma $\gamma$-glutamyltransferase levels.

Conclusions/interpretation This cross-sectional study supports the existence of a liver-alpha cell axis in humans: glucagon regulates plasma levels of amino acids, which in turn feedback to regulate the secretion of glucagon. With hepatic insulin resistance, reflecting hepatic steatosis, the feedback cycle is disrupted, leading to hyperaminoacidaemia and hyperglucagonaemia. The glucagon-alanine index is suggested as a relevant marker for hepatic glucagon signalling.
\end{abstract}

Keywords Amino acids · Glucagon · Insulin resistance $\cdot$ Liver damage

Nicolai J. Wewer Albrechtsen and Kristine Færch contributed equally to this study.

Jens J. Holst

jjholst@ sund.ku.dk

1 Department of Biomedical Sciences, Faculty of Health and Medical Sciences, University of Copenhagen, Blegdamsvej 3B, 2200 Copenhagen, Denmark

2 NNF Center for Basic Metabolic Research, Faculty of Health and Medical Sciences, University of Copenhagen, Copenhagen, Denmark

3 Steno Diabetes Center Copenhagen, Gentofte, Denmark
4 The Danish Diabetes Academy, Odense, Denmark

5 Department of Public Health, Section of General Practice, Aarhus University, Aarhus, Denmark

6 Center for Diabetes Research, Gentofte Hospital, University of Copenhagen, Hellerup, Denmark

7 Department of Clinical Medicine, Faculty of Health and Medical Sciences, University of Copenhagen, Copenhagen, Denmark

8 National Institute of Public Health, Southern Denmark University, Odense, Denmark 


\section{Research in context}

\section{What is already known about this subject?}

- Glucagon increases amino acid turnover in mice

- Amino acids regulate secretion of glucagon, and prolonged exposure results in alpha cell hyperplasia

- A liver-alpha cell axis seems to exist in rodents, which, when disrupted, impairs the effect of glucagon on amino acid turnover, leading to increased amino acid levels and hyperglucagonaemia

\section{What is the key question?}

- Does a similar liver-alpha cell axis exist in humans?

\section{What are the new findings?}

- In 1408 individuals with a wide range of blood glucose levels, glucagon and glucagonotropic amino acids were significantly associated

- Increasing liver fat (reflected by HOMA-IR) modified the association between glucagon and amino acids

- A glucagon-alanine index was developed and may serve as a marker of hepatic glucagon sensitivity

\section{How might this impact on clinical practice in the foreseeable future?}

- An increased glucagon-alanine index may indicate impaired hepatic glucagon sensitivity suggestive of impaired liver function

$\begin{array}{ll}\text { Abbreviations } \\ \text { ALT } & \text { Alanine aminotransferase } \\ \text { BCCA } & \text { Branched-chain amino acid } \\ \text { GGT } & \gamma \text {-Glutamyltransferase } \\ \text { ISI }_{0-120 m i n} & \begin{array}{l}\text { Peripheral insulin sensitivity as calculated } \\ \text { based on the first 120 min of OGTT }\end{array} \\ \text { NAFLD } & \text { Non-alcoholic fatty liver disease }\end{array}$

\section{Introduction}

Glucagon is an important glucose-regulatory hormone, which via a feedback cycle regulates hepatic glucose production, while the resulting glucose levels regulate the secretion of glucagon from the pancreatic alpha cells [1]. With the use of glucagon receptor antagonists in rodents and in individuals with type 2 diabetes, it has finally been agreed that inappropriate glucagon secretion makes an important contribution to hyperglycaemia in diabetes [2-4]. However, in addition to its glucose-regulatory effects, glucagon also regulates amino acid metabolism (ureagenesis) in the liver [5] and, conversely, amino acids stimulate the secretion of glucagon [6], forming a feedback loop between the liver and the pancreatic alpha cells [7, 8] - a liver-alpha cell axis. This glucagon-amino acid feedback loop may be as important for metabolism as the glucagon-glucose loop [9]. In line with this, individuals with complete disruption of glucagon signalling develop not hypoglycaemia but increasing amino acid levels, leading to severe alpha cell hypersecretion and hyperplasia $[7,10]$. In addition, hyperglucagonaemia (as in individuals with glucagon-producing tumours) does not always cause diabetes, but it severely decreases amino acid levels, resulting in muscular wasting and decreased cellular proliferation in the skin $[11,12]$.

Apart from these extremes, however, it is not clear how the feedback loop between amino acids and glucagon operates in healthy individuals, and in particular in individuals with impaired liver function, for example due to non-alcoholic fatty liver disease (NAFLD) [13]. Hypothetically, even slightly impaired liver function (appearing as hepatic insulin resistance) might attenuate glucagon-induced amino acid turnover (glucagon resistance), resulting in increased plasma levels of amino acids and hence increased glucagon secretion. In order to address this hypothesis, we studied whether fasting plasma glucagon concentrations were associated with fasting plasma concentrations of branched-chain amino acids (BCAAs) as well as four non-BCAAs (alanine, histidine, tyrosine and glutamine) and phenylalanine in a cross-sectional study including 1408 individuals with progressive stages of risk of type 2 diabetes. Furthermore, we examined whether hepatic insulin resistance (reflected by an increased HOMA-IR [14]) would modify glucagon-induced amino acid turnover.

\section{Methods}

Study population The study was based on data from the Danish ADDITION-PRO study [15], a risk-stratified cohort 
of individuals with low to high risk of developing type 2 diabetes that is nested within the ADDITION-Denmark study [16]. Individuals with impaired glucose regulation as well as a random subsample of individuals found at the ADDITIONDenmark screening (2001-2006) to have a lower risk of diabetes were invited to a follow-up health examination (20092011), and 2082 participants (50\% of those invited) attended [15]. The study was approved by the Ethics Committee of the Central Denmark Region (reference no. 20080229) and was conducted in accordance with the Declaration of Helsinki. All participants provided oral and written informed consent before participating in the study.

Examination and measurements Full details on the health examination and measurements as part of the ADDITIONPRO study have previously been described [15]. In brief, at the examination in 2009-2011, participants without known diabetes underwent a standard $75 \mathrm{~g}$ OGTT after an overnight fast of $8 \mathrm{~h}$ or longer. Blood samples were drawn at 0,30 and $120 \mathrm{~min}$ to assess serum concentrations of insulin, and plasma concentrations of glucose, glucagon and amino acids. Using a Tanita Body Composition Analyser (Tokyo, Japan), body weight was measured to the nearest $0.1 \mathrm{~kg}$ with participants wearing light indoor clothing without shoes, and height was measured to the nearest millimetre using a fixed rigid stadiometer (Seca, Hamburg, Germany). As this was a secondary analysis, we included data on metabolites that had already been measured, but also included new measurements of liver enzymes and urea.

Plasma alanine aminotransferase (ALT) and plasma glucose concentrations were determined using the Hitachi 912 system (Roche Diagnostics, Mannheim, Germany) or the Vitros 5600 system (Ortho Clinical Diagnostics, Illkirch, France). Values measured by the Vitros 5600 system were converted to correspond to values from the Hitachi 912 system using a validated regression equation $[15,17]$.

Serum insulin concentrations were measured by immunoassay (AutoDELFIA; PerkinElmer, Waltham, MA, USA). Blood samples for the measurement of glucagon, urea and $\gamma$-glutamyltransferase (GGT) were obtained in tubes containing EDTA, immediately put on ice and centrifuged, and the plasma was stored at $-80^{\circ} \mathrm{C}$.

Radio-immunological determinations of glucagon were performed as previously described [18] using a C-terminusspecific antibody (codename 4305), which reliably measures pancreatic glucagon as validated by sandwich ELISA and mass spectrometry [19]. The analytical detection limit was $1 \mathrm{pmol} / \mathrm{l}$, and the intra-assay and inter-assay $\mathrm{CVs}$ were $<6 \%$ and $<15 \%$, respectively. All samples for determination of glucagon were analysed consecutively over 2 months using identical quality controls and identical batches of all reagents.

A targeted NMR spectroscopy-based approach was used to measure plasma amino acid levels. This method, including
CVs, has previously been described in detail [20]. A total of four non-BCAAs (alanine, histidine, tyrosine and glutamine), phenylalanine and three BCAAs (isoleucine, leucine and valine) were measured. We calculated 'total non-BCAA' as the sum of the concentrations of alanine, histidine, tyrosine and glutamine, and 'total BCAA' as the sum of isoleucine, leucine and valine (not including phenylalanine).

GGT and urea were measured in plasma on a Cobas 8000 instrument, c802 module (Roche, Mannheim, Germany) using Cobas calibrators and reagents according to the manufacturer's instructions.

Calculations and statistical analyses Participants with known diabetes $(n=336)$ and those who had fasted for less than $8 \mathrm{~h}$ prior to the health examination $(n=20)$ were excluded from the analysis. We further excluded participants who had not had blood samples taken for measurement of plasma glucagon $(n=281)$, those in whom no amino acids were measured $(n=$ $26)$ and those without data on fasting serum insulin $(n=11)$, leaving 1408 (68\%) individuals for analysis.

Insulin resistance was calculated according to the HOMAIR [21]. As the model is based on glucose and insulin concentrations measured in the fasting state, HOMA-IR predominantly reflects hepatic insulin resistance $[14,22]$. The HOMA-IR values for the study population are presented as tertiles of HOMA-IR: lower tertile, 0.10-1.06; middle tertile, 1.07-1.84; upper tertile, 1.85-24.05.

Plasma concentrations of glucagon and alanine during the OGTT are presented as geometric means. Associations between fasting plasma glucagon (exposure) and fasting plasma levels of amino acids (outcome) were assessed using linear regression analysis. All analyses were adjusted for age and sex (model 1). We further adjusted the analyses for BMI (model 2) and HOMA-IR (model 3). In model 3, we first tested for deviation from linearity by including a quadratic term of glucagon. We next tested for a modifying effect of HOMA-IR on the associations between plasma amino acids and plasma glucagon concentrations. In case of a modifying effect of HOMA-IR, the associations are shown for the median, lower quartile and upper quartile of HOMA-IR. In a sensitivity analysis, we substituted, in model 3, HOMA-IR with peripheral insulin sensitivity (calculated based on the first $120 \mathrm{~min}$ of OGTT [ISI $\left.\mathrm{IL}_{0-120 \mathrm{~min}}\right]$ ), which is an estimate of whole-body/peripheral insulin sensitivity [23].

To facilitate direct comparisons of the strength of association between the eight amino acids and fasting glucagon, plasma levels of amino acids were standardised prior to analysis. The 1408 participants had complete data on all eight amino acids. Fasting glucagon was $\log _{2}$-transformed prior to analysis because the requirement for a normal distribution of model residuals was not met.

Because of the possible bidirectional relationship between amino acids and glucagon, we also performed analyses with 
fasting plasma glucagon as outcome and the different amino acids as explanatory factors in linear regression models adjusted for age, sex and HOMA-IR. Additionally, we combined the eight measured amino acids in order to assess the proportion of residual variance in fasting plasma glucagon explained by these amino acids after adjustment for age and sex. For comparison, we also calculated the proportion explained by HOMA-IR and by the amino acids in combination with HOMA-IR.

Finally, to generate a potential surrogate marker for the hepatic actions of glucagon on ureagenesis, we calculated a glucagon-alanine index using the following formula:

$$
\begin{aligned}
\text { glucagon }- \text { alanine index } & =\text { fasting plasma glucagon }(\mathrm{pmol} / \mathrm{l}) \\
& \times \text { fasting plasma alanine }(\mathrm{mmol} / \mathrm{l})
\end{aligned}
$$

Scatter plots of fasting concentrations of plasma glucagon and alanine were plotted, together with estimated levels of the new index for tertiles of HOMA-IR, plasma ALT and GGT. In addition, linear regression analysis adjusting for age and sex was used to assess the associations between the new index and HOMA-IR, ALT and GGT in separate analyses. Again, because the data did not meet the requirement for a normal distribution of model residuals, HOMA-IR, ALT and GGT were $\log _{\mathrm{e}}$-transformed prior to analysis, and, similarly, the glucagon-alanine index was $\log _{2}$-transformed.

Statistical analyses were performed in $\mathrm{R}$ version 3.2.3 ( $\mathrm{R}$ Foundation for Statistical Computing, www.R-project.org) and SAS version 9.4 (SAS Institute, Cary, NC, USA).

\section{Results}

Characteristics of the study participants The characteristics of the 1408 study participants according to tertiles of insulin resistance (HOMA-IR) are presented in Table 1. The mean fasting plasma glucagon concentration was significantly higher in individuals in the upper tertile compared with those in the middle and lower tertiles. Higher mean plasma levels of all BCAAs and alanine, tyrosine and glutamine, but not histidine, were also observed in individuals with higher levels of HOMA-IR.

HOMA-IR modifies circulating glucagon and alanine responses during an OGTT Plasma concentrations of glucagon and alanine decreased during the OGTT (Fig. 1a, b); however, the absolute concentrations of alanine differed by tertile of HOMA-IR. The $75 \mathrm{~g}$ glucose intake did not significantly affect the plasma concentration of alanine for individuals in the lower tertile of HOMA-IR (fasting: $0.26 \pm 0.06 \mathrm{mmol} / \mathrm{l}$; change in alanine $120 \mathrm{~min}$ after the OGTT: $-0.003 \mathrm{mmol} / \mathrm{l}$ [95\% CI $-0.007,0.000 \mathrm{mmol} / \mathrm{l}], p=0.076)$. However, for the middle and upper tertiles of HOMA-IR, fasting plasma concentrations of alanine were higher $(0.27 \pm 0.06$ and $0.28 \pm$ $0.06 \mathrm{mmol} / \mathrm{l}$, respectively), and in these two tertiles alanine concentration decreased in response to glucose intake (change in alanine $120 \mathrm{~min}$ after the OGTT: -0.010 [95\% CI -0.014 , $-0.007]$ and $-0.018 \mathrm{mmol} / 1[95 \% \mathrm{CI}-0.022,-0.015 \mathrm{mmol} / 1]$, respectively; $p<0.001$ for both) (Fig. 1b).

A proposed index for estimating the hepatic actions of glucagon Tertiles of HOMA-IR were significantly associated with higher levels of the glucagon-alanine index (Fig. 1c; $p<0.001$ ), with the highest levels of the index in the upper tertiles. The glucagon-alanine index also differed significantly between the tertiles of ALT and GGT $(p<0.001)$, although the differences were small. In linear regression analyses, we observed that a doubling of the glucagon-alanine index was associated with a $30 \%\left(95 \%\right.$ CI $\left.25 \%, 35 \% ; R^{2}=0.14\right)$ increase in HOMA-IR $(p<0.001)$, a $7 \%\left(95 \%\right.$ CI $\left.5 \%, 10 \% ; R^{2}=0.09\right)$ increase in plasma ALT $(p<0.001)$, and a $14 \%\left(95 \%\right.$ CI $\left.10 \%, 19 \% ; R^{2}=0.09\right)$ increase in GGT concentration after adjustment for age and sex.

The association between fasting plasma concentrations of glucagon and alanine is modified by HOMA-IR For phenylalanine, isoleucine, leucine and valine, we found statistically significant non-linear associations with fasting glucagon concentration $(p \leq 0.049)$. However, we found the deviations from linearity to be small and probably clinically irrelevant (data not shown), and therefore the following results are based on linear associations only.

For alanine, tyrosine, phenylalanine, 'total non-BCAA' (alanine, tyrosine, histidine and glutamine), isoleucine, leucine and total BCAA, we found a modifying effect of hepatic insulin resistance on the associations with fasting plasma glucagon $(p \leq 0.040)$. Hence, an interaction between fasting plasma glucagon and HOMA-IR was included in the models for these amino acids, and results are shown for different levels of HOMA-IR (the median and interquartile limits). As the estimated effects of glucagon were similar for models $1-3$, only results from the fully adjusted model are shown (model 3; Fig. 2).

The standardised effects on fasting plasma amino acids levels from increasing fasting plasma glucagon levels were comparable for alanine, tyrosine, isoleucine, leucine and valine (Fig. 2), whereas histidine and glutamine were not significantly associated with fasting glucagon levels ( $p \geq 0.127)$.

For the two non-BCAAs alanine and tyrosine a twofold increase in plasma glucagon was significantly associated with lower plasma concentrations of the amino acids. These inverse associations of glucagon with alanine and tyrosine were significantly impaired by increasing levels of HOMA-IR ( $p \leq$ 0.025) (Fig. 2a).

In contrast to the findings for the non-BCAAs, glucagon concentrations were significantly associated with higher plasma concentrations of the three BCAAs (isoleucine, leucine 
Table 1 Characteristics of the study participants in the ADDITION-PRO cohort by tertiles of insulin resistance (HOMA-IR)

\begin{tabular}{|c|c|c|c|c|}
\hline Variable & $\begin{array}{l}\text { Lower tertile } \\
(0.10-1.06)\end{array}$ & $\begin{array}{l}\text { Middle tertile } \\
(1.07-1.84)\end{array}$ & $\begin{array}{l}\text { Upper tertile } \\
(1.85-24.05)\end{array}$ & $p$ value $^{\mathrm{a}}$ \\
\hline$n$ & 469 & 470 & 469 & \\
\hline Age (years) & $66.8(7.4)$ & $65.9(7.0)$ & $66.0(6.7)$ & 0.073 \\
\hline Women $(\%)$ & $49.7(45.1,54.3)$ & $50.6(46.0,55.2)$ & $40.7(36.2,45.3)^{* \dagger}$ & 0.003 \\
\hline BMI $\left(\mathrm{kg} / \mathrm{m}^{2}\right)$ & $24.4(3.2)$ & $26.8(3.8)^{*}$ & $29.9(4.6)^{* \dagger}$ & $<0.001$ \\
\hline $\mathrm{HbA}_{1 \mathrm{c}}(\mathrm{mmol} / \mathrm{mol})$ & $43.3(4.0)$ & $43.9(4.0)$ & $46.1(6.0)^{* \dagger}$ & $<0.001$ \\
\hline $\mathrm{HbA}_{1 \mathrm{c}}(\%)$ & $5.6(0.3)$ & $5.6(0.3)$ & $5.8(0.5)^{* \dagger}$ & $<0.001$ \\
\hline Fasting plasma glucose (mmol/l) & $5.6(0.5)$ & $5.9(0.5)^{*}$ & $6.5(0.9)^{* \dagger}$ & $<0.001$ \\
\hline $120 \mathrm{~min}$ plasma glucose $(\mathrm{mmol} / \mathrm{l})$ & $5.9(1.7)$ & $6.5(1.8)^{*}$ & $8.0(2.8)^{*}$ & $<0.001$ \\
\hline Fasting serum insulin (pmol/l) & $21.0(17.0-25.0)$ & $37.0(32.0-42.0)^{*}$ & $69.0(55.0-90.0)^{* \dagger}$ & $<0.001$ \\
\hline HOMA-IR & $0.77(0.59-0.91)$ & $1.39(1.22-1.60)^{*}$ & $2.75(2.25-3.77)^{* \dagger}$ & $<0.001$ \\
\hline НОМА- $\beta$ & $28.0(22.1-35.0)$ & $44.4(35.6-54.7)^{\dagger}$ & $73.1(54.9-94.1)^{* \dagger}$ & $<0.001$ \\
\hline Fasting plasma glucagon (pmol/l) & $8.0(6.0-11.0)$ & $9.0(6.0-12.0)$ & $13.0(9.0-17.0)^{* \dagger}$ & $<0.001$ \\
\hline Glucagon-alanine index & $2.1(1.5-2.9)$ & $2.3(1.6-3.2)^{*}$ & $3.5(2.5-5.2)^{* \dagger}$ & $<0.001$ \\
\hline GGT (U/l) & $22.0(16.0-33.0)$ & $25.0(17.0-40.0)^{*}$ & $35.0(23.0-57.0)^{* \dagger}$ & $<0.001$ \\
\hline Plasma alanine (mmol/l) & $0.26(0.06)$ & $0.27(0.06)^{*}$ & $0.28(0.06)^{* \dagger}$ & $<0.001$ \\
\hline Plasma histidine (mmol/l) & $0.068(0.011)$ & $0.066(0.012)$ & $0.067(0.024)$ & 0.264 \\
\hline Plasma tyrosine $(\mathrm{mmol} / \mathrm{l})$ & $0.059(0.010)$ & $0.060(0.011)$ & $0.065(0.011)^{* \dagger}$ & $<0.001$ \\
\hline Plasma glutamine (mmol/1) & $0.059(0.010)$ & $0.060(0.011)$ & $0.065(0.011)^{* \dagger}$ & $<0.001$ \\
\hline Plasma phenyl alanine (mmol/l) & $0.038(0.005)$ & $0.038(0.006)$ & $0.040(0.006)^{* \dagger}$ & $<0.001$ \\
\hline Plasma isoleucine $(\mathrm{mmol} / \mathrm{l})$ & $0.038(0.011)$ & $0.041(0.014)^{*}$ & $0.049(0.016)^{*^{\dagger}}$ & $<0.001$ \\
\hline Plasma leucine (mmol/l) & $0.050(0.010)$ & $0.050(0.012)$ & $0.055(0.013)^{* \dagger}$ & $<0.001$ \\
\hline Plasma valine (mmol/l) & $0.14(0.03)$ & $0.15(0.03)$ & $0.16(0.03)^{* \dagger}$ & $<0.001$ \\
\hline Urea $(\mathrm{mmol} / \mathrm{l})$ & $5.5(1.5)$ & $5.5(1.7)$ & $5.7(2.2)$ & 0.086 \\
\hline
\end{tabular}

Data are shown as means (SD), medians (interquartile range) or percentages (95\% CI)

${ }^{\text {a }}$ Level of significance for the overall test of difference between groups of tertiles of insulin resistance ${ }^{*} p<0.05$ vs lower tertile; ${ }^{\dagger} p<0.05$ vs middle tertile and valine) and phenylalanine, and these associations were amplified by increasing levels of HOMA-IR, except for valine $(p \leq 0.040)$ (Fig. 2b).
In the sensitivity analysis substituting HOMA-IR with ISI $_{0-120 \min }$, we found no modifying effect of ISI $_{0-120 \min }$ on the association between plasma glucagon and plasma

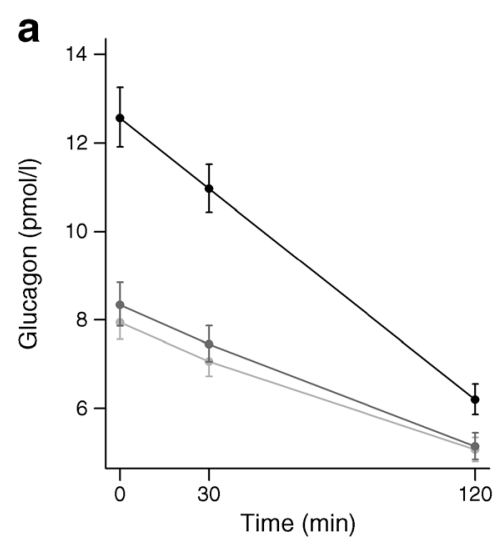

Fig. 1 Plasma concentrations of glucagon and alanine may reflect fasting HOMA-IR. (a) Plasma glucagon and (b) alanine concentrations during an OGTT by tertiles of HOMA-IR: lower tertile (light grey), middle tertile (dark grey) and upper tertile (black). Data are geometric means with $95 \%$ CI for glucagon, and means with $95 \%$ CI for alanine. (c) Scatter plots of fasting plasma glucagon as a function of fasting plasma alanine,
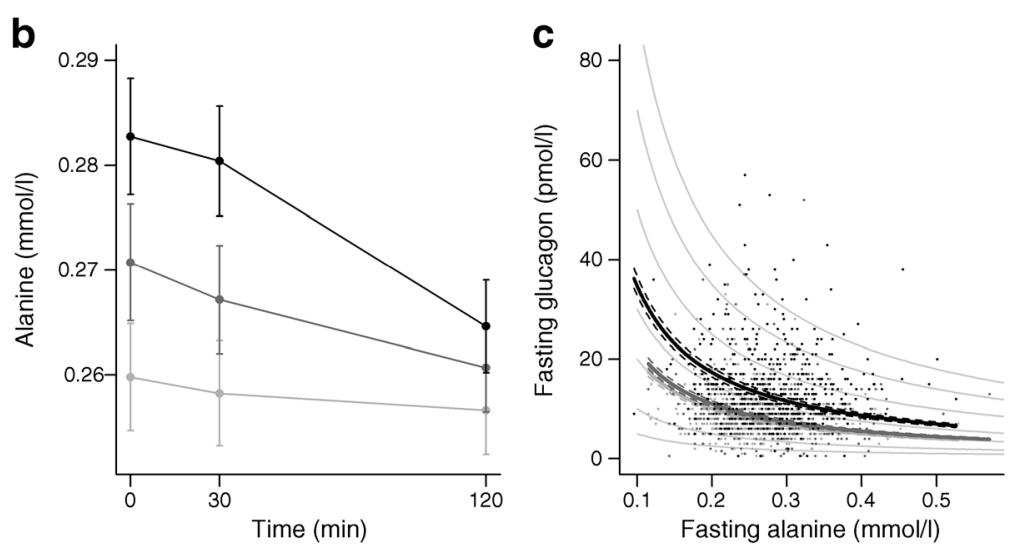

stratified by tertiles of HOMA-IR. Curves with $95 \%$ CI (dotted lines) are plotted within the range of observed fasting plasma alanine and shown for the lower tertile (thick light grey line), middle tertile (thick dark grey line) and upper tertile (thick black) line. The thin grey lines illustrate different levels of the (multiplicative) product: fasting plasma glucagon $\times$ fasting plasma alanine (hyperbolic function) 
a

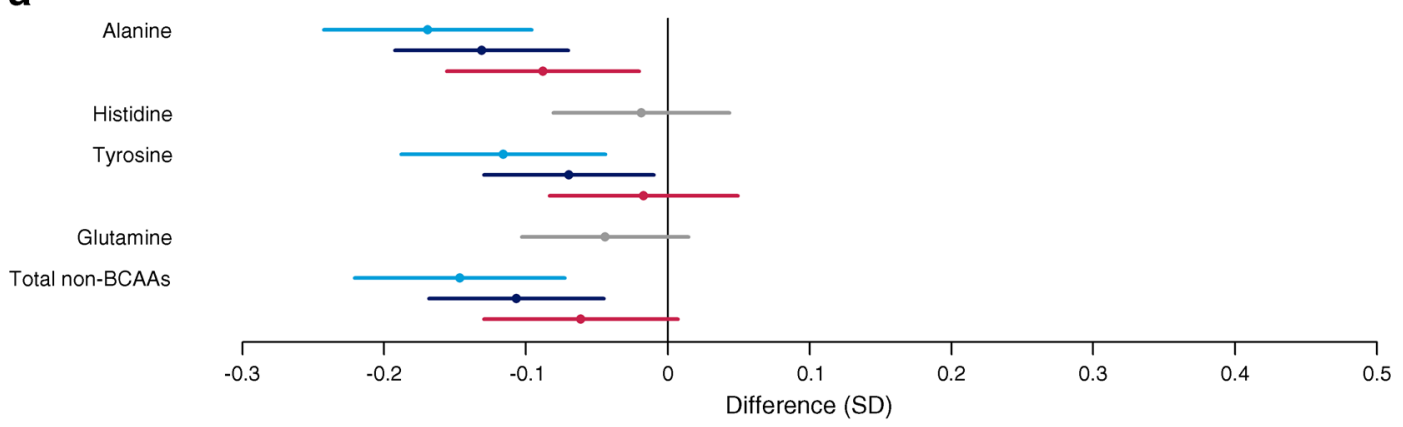

b

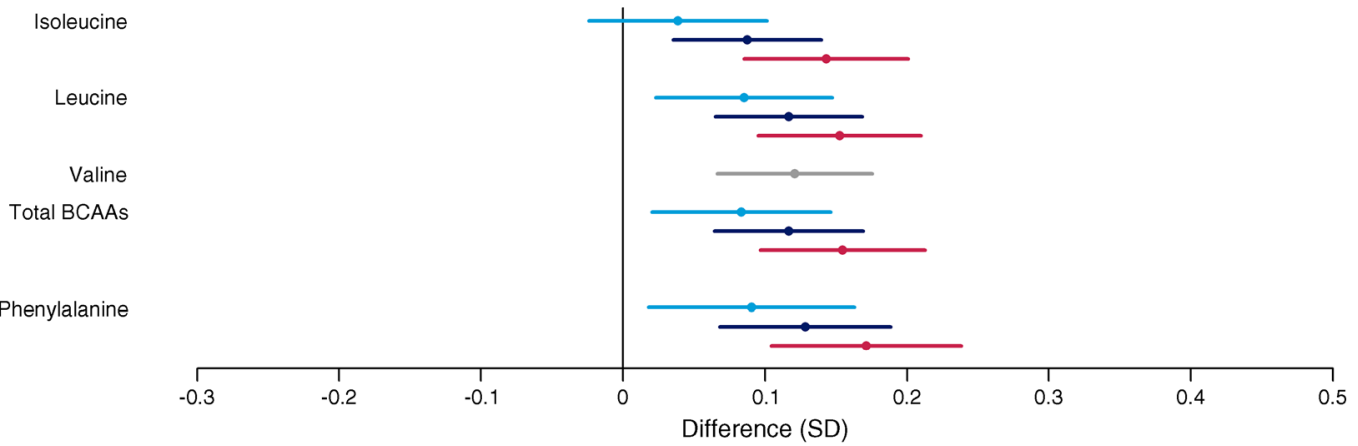

C

Urea

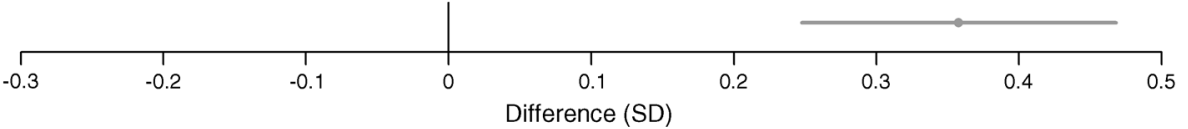

Fig. 2 Differential effect of glucagon on non-BCAAs and BCAAs. Difference (SD units) in plasma concentrations of (a) non-BCAAs, (b) BCAAs and (c) urea by a doubling of fasting plasma glucagon, adjusting for age, sex, BMI and insulin resistance (HOMA-IR). For plasma concentrations of alanine, tyrosine, total non-BCAAs, isoleucine, leucine, total BCAAs and phenylalanine, results are shown for three different levels of HOMA-IR: median quartile, dark blue (1.39); lower quartile, light blue (0.91); upper quartile, red (2.25). For histidine, glutamine, valine and urea, results are shown as a grey line. Total non-BCAAs is defined as the sum of alanine, histidine, tyrosine and glutamine concentrations. Total BCAAs is defined as the sum of isoleucine, leucine and valine (and does not include phenylalanine) concentrations of 'non-BCAAs' ( $p>0.166$ for all), but ISI $_{0-120 \min }$ modified the association between glucagon and total BCAAs $(p=0.036$ ), with decreasing effect size of glucagon for higher levels of ISI $_{0-120 \mathrm{~min}}$. Assuming that ureagenesis would be the outcome of glucagon action on amino acid metabolism, we also measured plasma urea levels in all samples. The results showed that a twofold increase in plasma glucagon was associated with a significant increase in plasma urea $(p<0.001)$; however, HOMA-IR did not have a significantly modifying effect ( $p=0.621$ ) (Fig. 2 c).

\section{Plasma amino acids and insulin resistance explain the vari-} ance in fasting plasma glucagon concentration As shown in Fig. 3, the eight amino acids and HOMA-IR explained an equal proportion (approximately 10\%) of the total residual variance in fasting plasma glucagon, but there was also an overlap due to an association between HOMA-IR and plasma amino acid concentrations, supporting the relationship between liver function, amino acids and glucagon secretion.

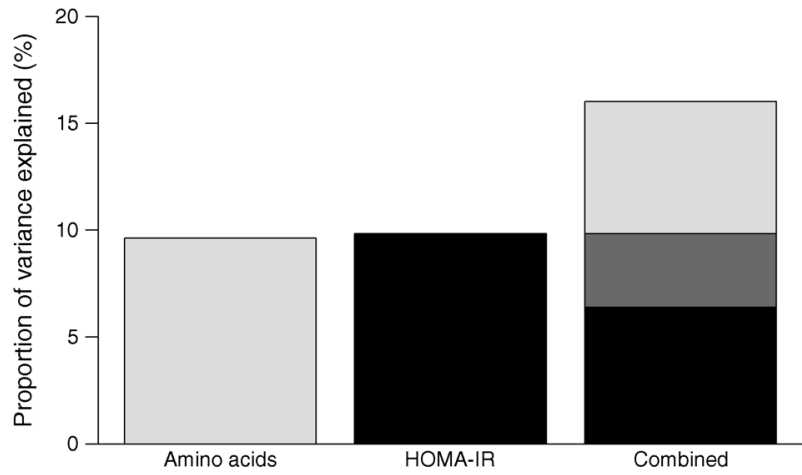

Fig. 3 Plasma concentrations of amino acids may explain variance in plasma glucagon concentrations. Proportion of variance in fasting plasma glucagon explained by all eight plasma amino acids, HOMA-IR, and plasma amino acids and HOMA-IR combined. In the 'Combined' bar, the light grey and black areas indicate the proportion of variance explained independently by plasma concentrations of amino acids and HOMA-IR, respectively, whilst the dark grey area indicates the proportion of variance explained by either HOMA-IR or plasma amino acids concentration owing to the correlation between them 


\section{Discussion}

The data presented here support that a feedback loop exists in humans between glucagon and certain glucagonotropic (alanine, tyrosine and glutamine) amino acids, and when this is disrupted due to hepatic abnormalities (reflected here as elevated HOMA-IR levels), the lowering effect of glucagon on the plasma concentration of amino acids may be gradually impaired. This leads to increased amino acid concentrations, and eventually increased glucagon concentrations, as illustrated by increases in the new, proposed 'glucagon-alanine index'. Specifically, we found in 1408 individuals with normal and impaired glucose regulation that the fasting plasma levels of four glucagonotropic amino acids were inversely associated with fasting glucagon levels independent of age, sex, BMI and peripheral insulin resistance (ISI ${ }_{0-120}$ ), and that the associations were modified by hepatic insulin resistance (HOMAIR). Plasma concentrations of both glucagon and nonBCCAs (e.g. alanine) during an OGTT were affected by increasing HOMA-IR; the BCAAs were also affected but in the opposite direction, as previously reported [24-26]. These observations support our hypothesis that impaired hepatic glucagon signalling, potentially due to fat accumulation in the liver and resulting hepatic insulin resistance [14], impairs glucagon's ability to lower plasma levels of non-BCAAs. Taken together, the current findings support the proposed existence and importance of a liver-alpha cell axis in humans [8].

Alanine and other non-BCAAs, including tyrosine and glutamine, have previously been shown to increase glucagon secretion in humans and dogs, whereas this is not the case for BCAAs [27, 28]. In addition, prolonged administration of non-BCAAs to rodents results in alpha cell hyperplasia and hyperglucagonaemia $[7,29]$. These observations have led to the proposal of the existence of a hitherto neglected feedback loop between the pancreatic alpha cells and the liver $[8,30]$. Consistent with this proposed feedback loop, individuals with glucagon-producing tumours have decreased levels of plasma amino acids [11,31], and, conversely, individuals with glucagon receptor mutations $[10,32,33]$ and mice with glucagon receptor deficiency $[30,34]$ exhibit increased levels of plasma amino acids. Hyperglucagonaemia has also been reported in individuals with fatty liver disease independent of their glycaemic status [35], and this has recently been linked to an increased plasma pool of amino acids including alanine, but excluding the BCAAs isoleucine, leucine and valine [36].

In this study, only eight amino acids, including three BCAAs, were measured, which obviously limits the interpretation of our results. Measurement of all circulating amino acids would have been ideal but was unfeasible based on the current NMR-based platform. We may therefore have underor overestimated the association between glucagon and several of the additional non-BCAAs. Glutamine, although known to spontaneously degrade in solution [37], was included in the study because in particular this amino acid has been suggested to be of importance for amino acid-induced alpha cell proliferation [7] and glucagon secretion [38]. The importance of the measured amino acids for determining plasma levels of glucagon clearly needs further investigation given that only around $10 \%$ of the variance in plasma glucagon could be accounted for. Therefore, our results cannot be extrapolated to general hyperaminoacidaemia, but they do show that a link seems to exist between certain glucagonotropic amino acids and plasma levels of glucagon. Indeed, the non-BCAAs included in our analysis have recently been linked to alpha cell proliferation in animal models [39, 40]. Assuming that ureagenesis would be the outcome of glucagon action on amino acid metabolism, we also measured plasma urea levels in all samples. The results showed that a twofold increase in plasma glucagon was associated with a significant increase in plasma urea, therefore further supporting the hepatic actions of glucagon on amino acid metabolism. Finally, as the data shown here rely on a cross-sectional study design, it is important to note that causality cannot be implied.

In addition, disturbances of glucagon secretion at fasting or during intake of carbohydrates may be related to imbalances of insulin secretion and insulin sensitivity [41, 42], the latter having been associated with increased plasma concentrations of BCAAs and phenylalanine [26, 43]. This may also explain why we observed increasing plasma concentrations of BCAAs with increasing plasma concentrations of glucagon, and may reflect a glucose-related interaction of glucagon and insulin, as recently reported [44].

Interestingly, we recently demonstrated that glucose regulation during development of insulin resistance was associated not also with hypersecretion of insulin, but also with a reduced ability to acutely suppress glucagon after glucose intake [45]. The hypersecretion of glucagon from the pancreatic alpha cells may stem from an impairment of hepatic glucagon signalling (potentially caused by excessive food intake that may result in NAFLD and insulin resistance), which then, due to decreased glucagon-induced amino acid turnover, would result in hyperaminoacidaemia [36]. Finally, the hyperaminoacidaemia, and in particular the increased plasma concentrations of the glucagon-stimulatory amino acids [27, 28] alanine and tyrosine, may, as suggested by Solloway et al [7], result in hyperglucagonaemia, counteracting the NAFLDinduced impairment of glucagon-induced ureagenesis. Indeed, in the current study we found that elevations in plasma glucagon concentrations were associated with increased ureagenesis, as reflected by increased plasma concentrations of urea. However, this association was not significantly modified by HOMA-IR.

A major strength of this study is the large number of study participants encompassing a wide range of glycaemia from normal glucose tolerance to screen-detected and, hence, untreated type 2 diabetes. Importantly the measurement of 
glucagon, which is demanding when it comes to analysing the subtle effects of small changes in fasting glycaemia on glucagon concentration, was carried out using a validated, highly sensitive radioimmunoassay, thus diminishing the risk of including erroneous glucagon measures, as may occur with some commercial glucagon assays [46]. Because of the size of the study, hepatic insulin resistance was evaluated by the relatively simple HOMA-IR model, which is often used in epidemiological studies, and not by hyperinsulinaemiceuglycaemic clamp, which is the gold standard in smaller but in-depth physiological studies. Nevertheless, HOMA-IR shows a fairly good correlation with hepatic insulin resistance measured by hyperinsulinaemic-euglycaemic clamp combined with tracer techniques to estimate hepatic glucose production [22], and is therefore a useful surrogate marker of hepatic insulin resistance. Moreover, we did not observe the same modifying effect of ISI $_{0-120}$ as of HOMA-IR on the associations between fasting plasma levels of glucagon and non-BCAAs, which supports the notion of HOMA-IR being mainly related to insulin resistance in the liver. In addition, GGT, a liver-related enzyme that contributes to the transfer of $\gamma$-glutamyl groups to other amino acids and which has been suggested to be a potential surrogate marker of hepatic steatosis [47], was here coupled to changes in plasma glucagon and HOMA-IR, thereby supporting the suggestion that the increases in glucagon concentrations might be due to increased hepatic fat. HOMA-IR was recently demonstrated to be closely linked to hepatic steatosis [14].

Taken together, our findings suggest that hyperglucagonaemia may reflect disturbances in the physiological feedback loop between amino acid metabolism and the pancreatic alpha cells, rather than abnormalities of glucose metabolism. Observations of elevated fasting concentrations of plasma glucagon levels in humans should therefore direct attention to the functional status of the liver. Since hyperglucagonaemia is likely to be coupled to impaired amino acid metabolism, a combination of these two entities in the form of a product is likely to be a more sensitive measure of the abnormality than is either variable alone. The proposed glucagon-alanine index may provide an early indication of any abnormality of the feedback cycle. Whereas failures of alpha cell secretion are not well characterised, disturbances of liver function are common. However, based on our novel findings and partly as a consequence of improved assays for glucagon measurement [48], disturbances in alpha cell secretion may now begin to be uncovered. The usefulness of exploring such defects by use of our proposed glucagon-alanine index needs further studies. For instance, it will be interesting to see whether changes in hepatic insulin and glucagon sensitivity (HOMAIR and the glucagon-alanine index) move in parallel or follow distinct trajectories over time. Moreover, recent research has suggested that disruption of the glucagon-liver cycle (with glucagon receptor antagonists) may also lead to disturbances in lipid metabolism [49]. This has not been addressed in the present study, but needs to be investigated.

In conclusion, higher fasting plasma glucagon concentrations were associated with lower concentrations of certain non-BCAAs including alanine, tyrosine and glutamine, and with higher concentrations of BCAAs, in a large study population covering a broad glycaemic range from normal glucose tolerance to screen-detected type 2 diabetes. A liver-alpha cell axis may therefore exist in humans by which glucagon controls plasma levels of amino acid through hepatic gluconeogenesis and ureagenesis. Importantly, impaired liver function (increased HOMA-IR) weakened this relationship, resulting in higher amino acid levels and ensuing hyperglucagonaemia. If validated in other studies, a glucagon-alanine index may be a useful surrogate marker of glucagon receptor signalling in humans.

Acknowledgements The authors acknowledge the ADDITION-PRO study centres, the staff and the participants for their important contribution to the study. The authors thank laboratory technicians L. A. Albæk and S. P. O. Olesen, both at the Department of Endocrinology, University of Copenhagen, Denmark.

Data availability Authors can confirm that all relevant data are included in the article.

Funding The ADDITION-Denmark study was supported by the National Health Services in the counties of Copenhagen, Aarhus, Ringkøbing, Ribe and Southern Jutland in Denmark; the Danish Council for Strategic Research; the Danish Research Foundation for General Practice; Novo Nordisk Foundation; the Danish Centre for Evaluation and Health Technology Assessment; the Diabetes Fund of the National Board of Health; the Danish Medical Research Council; the Aarhus University Research Foundation; and the Danish Council for Strategic Research. The ADDITION-PRO study was funded by an unrestricted grant from the European Foundation for the Study of Diabetes/Pfizer for Research into cardiovascular disease risk reduction in individuals with diabetes (74550801). TL has received unrestricted grants for the ADDITION study from public foundations. NJWA was supported by the NNF Center for Basic Metabolic Research, University of Copenhagen, NNF application number: 13563 (Novo Nordisk Foundation, Denmark), EliteForsk Rejsestipendiat (2016), the Danish Council for Independent Research (DFF-1333-00206A), Augustinus Foundation, Aase og Ejnar Danielsens Fond, Mærsk Fonden, Holger Rabitz fond, Læge Johannes Nicolaj Krogsgaard og hustru Else Krogsgaards minde-legat for medicinsk forskning og medicinske studenter ved Københavns Universitet, the European Molecular Biology Organization (EMBO) and the European Foundation for the Study of Diabetes (EFSD). KF is supported by the Novo Nordisk Foundation. The Novo Nordisk Foundation Center for Basic Metabolic Research is an independent Research Center at the University of Copenhagen partially funded by an unrestricted donation from the Novo Nordisk Foundation (www.metabol.ku.dk). YM is funded by the Danish Diabetes Academy. AEJ is supported by the Danish Council for Independent Research, European Union, FP7, Marie Curie Actions, IEF and Lundbeck Foundation.

The study sponsor was not involved in the design of the study; the collection, analysis, and interpretation of data; writing the report; or the decision to submit the report for publication.

Duality of interest At the time of the initiation of the ADDITION-PRO study, the Steno Diabetes Center Copenhagen (SDCC) operated under the 
name Steno Diabetes Center A/S, owned by Novo Nordisk A/S, and worked as a research hospital in the Danish National Health Service. The ADDITION-PRO study received internal research and equipment funds from Steno Diabetes Center A/S. As of 1 January 2017, Steno Diabetes Center A/S formally and legally transitioned to the Capital Region of Copenhagen, a public non-profit body, to become the SDCC. The SDCC receives part of its core funding from unrestricted grants from the Novo Nordisk Foundation. NJWA and JJH have received speaker fees from MSD Denmark in relation to glucagon biology. KF, TMJ, MEJ and DV are employed by SDCC. KF, DRW, TMJ, SST, TL, OP, MEJ and DV hold shares in Novo Nordisk A/S. TL has received unrestricted grants between 2000 and 2011 for the ADDITION study (screening and intensive treatment of type 2 diabetes in primary care) from the medical industry: Novo Nordisk AS, Novo Nordisk Scandinavia AB, ASTRA Denmark, Pfizer Denmark, GlaxoSmithKline Pharma Denmark, SERVIER Denmark A/S and HemoCue Denmark A/S. All other authors declare that there is no duality of interest associated with their contribution to this manuscript.

Contribution statement NJWA and KF drafted the manuscript. NJWA, KF, TMJ, JP, DV and JJH researched and interpreted the data. DV performed the statistical analyses. DRW, TL, OP and TH designed the ADDITION-PRO study (the parts related to glucagon and metabolomics). DRW, YM, AEJ, KDG, MWS, SST, TL, OP, FKK, TH and MEJ contributed to interpretation of the data. TMJ, DRW, JP, YM, AEJ, KDG, MWS, SST, TL, OP, FKK, TH, MEJ, DV and JJH revised the manuscript critically. All authors approved the final version of manuscript. JJH and DV are the guarantors of this work and, as such, had full access to all the data in the study and take responsibility for the integrity of the data and the accuracy of the data analysis.

\section{References}

1. Gromada J, Franklin I, Wollheim CB (2007) $\alpha$-cells of the endocrine pancreas: 35 years of research but the enigma remains. Endocr Rev 28:84-116

2. Kazda CM, Garhyan P, Kelly RP et al (2015) A randomized, double-blind, placebo-controlled phase 2 study of the glucagon receptor antagonist LY2409021 in patients with type 2 diabetes. Diabetes Care 37:1241-1249

3. Petersen KF, Sullivan JT (2001) Effects of a novel glucagon receptor antagonist (Bay 27-9955) on glucagon-stimulated glucose production in humans. Diabetologia 44:2018-2024

4. Johnson DG, Goebel CU, Hruby VJ, Bregman MD, Trivedi D (1982) Hyperglycemia of diabetic rats decreased by a glucagon receptor antagonist. Science 215:1115-1116

5. Boden G, Rezvani I, Owen OE (1984) Effects of glucagon on plasma amino acids. J Clin Investig 73:785-793

6. Assan R, Marre M, Gormley M (1983) The amino acid-induced secretion of glucagon. In: Lefebvre PJ (ed) Glucagon II. Spinger, Heidelberg, pp 19-41

7. Solloway Mark J, Madjidi A, Gu C et al (2015) Glucagon couples hepatic amino acid catabolism to mTOR-dependent regulation of $\alpha$-cell mass. Cell Rep 12:495-510

8. Holst JJ, Wewer Albrechtsen NJ, Pedersen J, Knop FK (2017) Glucagon and amino acids are linked in a mutual feedback cycle: the liver-alpha-cell axis. Diabetes 66:235-240

9. Unger RH, Cherrington AD (2012) Glucagonocentric restructuring of diabetes: a pathophysiologic and therapeutic makeover. J Clin Investig 122:4-12

10. Larger E, Wewer Albrechtsen NJ, Hansen LH et al (2016) Pancreatic alpha-cell hyperplasia and hyperglucagonemia due to a glucagon receptor splice mutation. Endocrinol Diabetes Metab Case Rep 2016:16-0081

11. Norton JA, Kahn CR, Schiebinger R, Gorschboth C, Brennan MF (1979) Amino acid deficiency and the skin rash associated with glucagonoma. Ann Intern Med 91:213-215

12. Alexander EK, Robinson M, Staniec M, Dluhy RG (2002) Peripheral amino acid and fatty acid infusion for the treatment of necrolytic migratory erythema in the glucagonoma syndrome. Clin Endocrinol 57:827-831

13. Samuel VT, Liu ZX, Qu X et al (2004) Mechanism of hepatic insulin resistance in non-alcoholic fatty liver disease. J Biol Chem 279:32345-32353

14. Isokuortti E, Zhou Y, Peltonen M et al (2017) Use of HOMA-IR to diagnose non-alcoholic fatty liver disease: a population-based and inter-laboratory study. Diabetologia 60:1873-1882

15. Johansen NB, Hansen AL, Jensen TM et al (2012) Protocol for ADDITION-PRO: a longitudinal cohort study of the cardiovascular experience of individuals at high risk for diabetes recruited from Danish primary care. BMC Public Health 12:1078

16. Lauritzen T, Griffin S, Borch-Johnsen K, Wareham NJ, Wolffenbuttel BH, Rutten G (2000) The ADDITION study: proposed trial of the cost-effectiveness of an intensive multifactorial intervention on morbidity and mortality among people with type 2 diabetes detected by screening. Int J Obes Relat Metab Disord 24(Suppl 3):S6-S11

17. Færch K, Torekov SS, Vistisen D et al (2015) Glucagon-like peptide-1 (GLP-1) response to oral glucose is reduced in pre-diabetes, screen-detected type 2 diabetes and obesity, and influenced by sex: the ADDITION-PRO study. Diabetes 64:2513-2525

18. Wewer Albrechtsen NJ, Bak MJ, Hartmann B et al (2015) Stability of glucagon-like peptide-1 and glucagon in human plasma. Endocr Connect 4:50-57

19. Lund A, Bagger JI, Wewer Albrechtsen NJ et al (2015) Evidence of extrapancreatic glucagon secretion in man. Diabetes 65:585-597

20. Soininen P, Kangas AJ, Wurtz P et al (2009) High-throughput serum NMR metabonomics for cost-effective holistic studies on systemic metabolism. Analyst 134:1781-1785

21. Matthews DR, Hosker JP, Rudenski AS, Naylor BA, Treacher DF, Turner RC (1985) Homeostasis model assessment: insulin resistance and beta-cell function from fasting plasma glucose and insulin concentrations in man. Diabetologia 28:412-419

22. Abdul-Ghani MA, Matsuda M, Balas B, DeFronzo RA (2007) Muscle and liver insulin resistance indexes derived from the oral glucose tolerance test. Diabetes Care 30:89-94

23. Gutt M, Davis CL, Spitzer SB et al (2000) Validation of the insulin sensitivity index $\left(\mathrm{ISI}_{0,120}\right)$ : comparison with other measures. Diabetes Res Clin Pract 47:177-184

24. Yamada C, Kondo M, Kishimoto N et al (2015) Association between insulin resistance and plasma amino acid profile in nondiabetic Japanese subjects. J Diabetes Investig 6:408-415

25. Connelly MA, Wolak-Dinsmore J, Dullaart RPF (2017) Branched chain amino acids are associated with insulin resistance independent of leptin and adiponectin in subjects with varying degrees of glucose tolerance. Metab Syndr Relat Disord 15:183-186

26. Mahendran Y, Jonsson A, Have CT et al (2017) Genetic evidence of a causal effect of insulin resistance on branched-chain amino acid levels. Diabetologia 60:873-878

27. Muller WA, Faloona GR, Unger RH (1971) The effect of alanine on glucagon secretion. J Clin Invest 50:2215-2218

28. Rocha DM, Faloona GR, Unger RH (1972) Glucagon-stimulating activity of 20 amino acids in dogs. J Clin Invest 51:2346-2351

29. Galsgaard KD, Winther-Sorensen M, Orskov C et al (2017) Disruption of glucagon receptor signaling causes hyperaminoacidemia exposing a possible liver - alpha-cell axis. Am J Phys Endocrinol Metab. https:// doi.org/10.1152/ajpendo.00198.2017 
30. Longuet C, Robledo AM, Dean ED et al (2013) Liver-specific disruption of the murine glucagon receptor produces $\alpha$-cell hyperplasia: evidence for a circulating $\alpha$-cell growth factor. Diabetes 62: $1196-1120$

31. Almdal TP, Heindorff H, Bardram L, Vilstrup H (1990) Increased amino acid clearance and urea synthesis in a patient with glucagonoma. Gut 31:946-948

32. Zhou C, Dhall D, Nissen NN, Chen C-R, Yu R (2009) A homozygous P86S mutation of the human glucagon receptor is associated with hyperglucagonemia, $\alpha$ cell hyperplasia, and islet cell tumor. Pancreas 38:941-946

33. Miller HC, Kidd M, Modlin IM et al (2015) Glucagon receptor gene mutations with hyperglucagonemia but without the glucagonoma syndrome. World J Gastrointest Surg 7:60-66

34. Gelling RW, Du XQ, Dichmann DS et al (2003) Lower blood glucose, hyperglucagonemia, and pancreatic $\alpha$ cell hyperplasia in glucagon receptor knockout mice. Proc Natl Acad Sci U S A 100: $1438-1443$

35. Junker AE, Gluud L, Holst JJ, Knop FK, Vilsboll T (2016) Diabetic and nondiabetic patients with nonalcoholic fatty liver disease have an impaired incretin effect and fasting hyperglucagonaemia. J Intern Med 279:485-493

36. Wewer Albrechtsen NJ, Junker AE, Christensen M et al (2017) Hyperglucagonemia correlates with plasma levels of nonbranched chained amino acids in patients with liver disease independent of type 2 diabetes. Am J Physiol Gastrointest Liver Physiol. https://doi.org/10.1152/ajpgi.00216.2017

37. Khan K, Elia M (1991) Factors affecting the stability of Lglutamine in solution. Clin Nutr 10:186-192

38. Cabrera O, Jacques-Silva MC, Speier S et al (2008) Glutamate is a positive autocrine signal for glucagon release. Cell Metab $7: 545-554$
39. Dean ED, Li M, Prasad N et al (2017) Interrupted glucagon signaling reveals hepatic alpha cell axis and role for L-glutamine in $\alpha$ cell proliferation. Cell Metab 25:1362-1373

40. Kim J, Okamoto H, Huang $\mathrm{Z}$ et al (2017) Amino acid transporter Slc38a5 controls glucagon receptor inhibition-induced pancreatic $\alpha$ cell hyperplasia in mice. Cell Metab 25:1348-1361

41. Raskin P, Unger RH (1978) Hyperglucagonemia and its suppression. N Engl J Med 299:433-436

42. Ahren B (2006) Glucagon secretion in relation to insulin sensitivity in healthy subjects. Diabetologia 49:117-122

43. Newgard CB, An J, Bain JR et al (2009) A branched-chain amino acid-related metabolic signature that differentiates obese and lean humans and contributes to insulin resistance. Cell Metab 9:311-326

44. Holst JJ, Holland W, Gromada J et al (2017) Insulin and glucagon: partners for life. Endocrinology 158:696-701

45. Faerch K, Vistisen D, Pacini G et al (2016) Insulin resistance is accompanied by increased fasting glucagon and delayed glucagon suppression in individuals with normal and impaired glucose regulation. Diabetes 65:3473-3481

46. Wewer Albrechtsen NJ, Veedfald S, Plamboeck A et al (2016) Inability of some commercial assays to measure suppression of glucagon secretion. J Diabetes Res. https://doi.org/10.1155/2016/8352957

47. Dixon JB, Bhathal PS, O'Brien PE (2006) Weight loss and nonalcoholic fatty liver disease: falls in gamma-glutamyl transferase concentrations are associated with histologic improvement. Obes Surg 16:1278-1286

48. Wewer Albrechtsen N, Hartmann B, Veedfald S et al (2014) Hyperglucagonaemia analysed by glucagon sandwich ELISA: nonspecific interference or truly elevated levels? Diabetologia 57:1919-1926

49. Guzman CB, Zhang XM, Liu R et al (2017) Treatment with LY2409021, a glucagon receptor antagonist, increases liver fat in patients with type 2 diabetes. Diabetes Obes Metab 19:1521-1528 